\title{
钯催化芳基乙炔对氧/氮杂苯并降冰片烯开环反应研究
}

\author{
陈花䂞 \\ 李嗣锋 \\ 徐建斌 \\ 杨清镜 \\ 刘珊珊 \\ 周永云 黄超樊保敏* \\ (云南民族大学 民族药资源化学国家民委-教育部重点实验室 昆明 650500)
}

\begin{abstract}
摘要 过渡金属催化的碳亲核试剂对氧/氮杂苯并降冰片烯的开环反应是合成氢化菱衍生物的有效方法之一. 在该类 反应中，采用末端炔烃作为碳亲核试剂在反应操作、底物适用范围等多个方面都具有已报道的各种有机金属亲核试剂 所不具备的优势. 目前, 对末端炔烃与氧/氮杂苯并降冰片烯的开环反应的研究进展有限, 只有 $\mathrm{Ni}(\mathrm{dppe}) \mathrm{Cl}_{2} / \mathrm{ZnCl}_{2} / \mathrm{Zn}$ 和 手性的 $\mathrm{Rh}$ 两种催化体系被应用到该类反应当中，但都面临一些不足. 为该类反应开发简单有效的新型催化体系仍然具 有重要的意义和价值. 我们通过研究发现: $\mathrm{PdCl}_{2}$ 与 Xantphos 的配合物是芳基乙炔对氧/氮杂苯并降冰片烯亲核开环反 应的有效催化剂. 在优化后的反应条件下，该 Pd 催化剂具有良好的催化活性和底物适应能力，能够实现各种取代的芳 基乙炔对氧/氮杂苯并降冰片烯的亲核加成，并以较好的收率生成目标开环产物.

关键词 钯; 芳基乙炔; 氧杂苯并降冰片烯; 氮杂苯并降冰片烯; 开环反应
\end{abstract}

\section{Palladium-Catalyzed Ring-Opening Reaction of Oxa/Azabenzonorbornadienes with Aryl Acetylenes}

\author{
Chen, Hualei Li, Sifeng Xu, Jianbin Yang, Qingjing Liu, Shanshan \\ Zhou, Yongyun Huang, Chao Fan, Baomin* \\ (Key Laboratory of Chemistry in Ethnic Medicinal Resources, State Ethnic Affairs Commission \& Ministry of \\ Education, Yunnan University of Nationalities, Kunming 650500, China)
}

\begin{abstract}
The transition-metal catalyzed ring opening reaction of oxa/azabenzonorbornadienes with carbo-nucleophiles has played an important role in the synthesis of tetrahydronaphthalene structures, which widely exist in natural products and bioactive molecules. Caused by the easy manipulation of reaction procedures and the less limits of suitable substrates, the ring opening reaction of oxa/azabenzonorbornadienes with terminal alkynes as nucleophiles has more potential in organic synthesis than with the organometal nucleophiles, such as zinc reagents, lithium reagents, Grignard reagents, aluminium reagents, organoboronic acids and zirconium reagents. However, only limited progress had been achieved for the ring opening reaction of oxa/azabenzonorbornadienes with terminal alkynes. Up to now, only a $\mathrm{Ni}(\mathrm{dppe}) \mathrm{Cl}_{2} / \mathrm{ZnCl}_{2} / \mathrm{Zn}$ catalytic system and a chiral rhodium catalyst had been developed for this reaction. Unfortunately, the former formed a relatively complicated reaction system, and suffered from the absence of chiral control ability. The latter was generated by composing of $\left[\mathrm{Rh}(\mathrm{OAc})\left(\mathrm{C}_{2} \mathrm{H}_{4}\right)_{2}\right]_{2}$ with $(R)$-DTBM-segphos and could result in high enantioselectivity. But the scope of suitable substrates for it was too limited, and only sterically bulky acetylenes were found useful. Developing new and efficient catalysts for this reaction is still interesting and desirable. As the continuation of our study on transition-metal catalyzed reactions between norbornadienes and terminal alkynes, we surprisingly found that the complex of $\mathrm{PdCl}_{2}$ and Xantphos could catalyze the ring opening reaction of oxabenzonorbornadiene 1a with $p$-methoxyphenylacetylene 2a smoothly. The optimization of reaction conditions showed that 1,2-dichloroethane was the only effective solvent and $70{ }^{\circ} \mathrm{C}$ was the proper reaction temperature. The investigation on the scope of suitable substrates showed that this new developed $\mathrm{PdCl}_{2} / \mathrm{Xantphos}$ catalyst had good tolerance for various functional groups. Under the promotion of this Pd catalyst, a series of oxa/azabenzonorbornadienes could react with various aryl acetylenes, having different substituents on the phenyl rings, smoothly to generate the ring opening products in good yields. Further efforts to design and synthesize new Pd catalysts to realize the asymmetric version of the ring opening reaction of oxa/azabenzonorbornadienes with terminal alkynes are still in progress.
\end{abstract}

Keywords palladium; aryl acetylene; oxabenzonorbornadiene; azabenzonorbornadiene; ring-opening reaction

\footnotetext{
* E-mail: adams.bmf@hotmail.com; Fax: 0086-0871-65913103.

Received May 20, 2013; published June 27, 2013.

Supporting information for this article is available free of charge via the Internet at http://sioc-journal.cn.

Project supported by the National Natural Science Foundation of China (No. 21162040, 20902080, 21362043) and the Applied Basic Research Program of Yunnan Province (No. 2012FB170).

项目受国家自然科学基金(No. 21162040, 20902080, 21362043)和云南省应用基础研究计划项目(No. 2012FB170)资助。
} 


\section{1 引言}

氢化荎结构单元广泛存在于具有生物活性的天然 产物及药物分子当中 ${ }^{[1]}$, 相应的合成研究也因此受到广 泛关注. 过渡金属催化的碳亲核试剂对氧/氮杂苯并降 冰片烯的开环反应 ${ }^{[2]}$ 就是近年来发展起来的构筑氢化荎 结构单元的重要方法之一. 在过去的研究中, 对空气、 水等较敏感且亲核性较强的有机金属试剂是最常用的 碳亲核试剂. 如在铑、铜、镍等过渡金属催化剂的催化 下, 有机锂 ${ }^{[3]}$ 、有机锌 ${ }^{[4]}$ 、有机镁 $(\text { 格式试剂 })^{[5]}$ 、有机 铝 ${ }^{[6]}$ 、有机锆 ${ }^{[7]}$ 等金属试剂能够有效地对氧/氮杂苯并降 冰片烯进行亲核开环, 并以较好的收率得到相应的氢化 菜衍生物. 但这些亲核开环反应需要在无水、无氧的条 件下才能有效进行. 虽然采用对水和空气不敏感的有机 嗍酸试剂做亲核试剂也能够实现对氧/氮杂苯并降冰片 烯的开环反应, 但往往只有采用芳基硼酸试剂才能给出 好的结果 ${ }^{[8]}$.
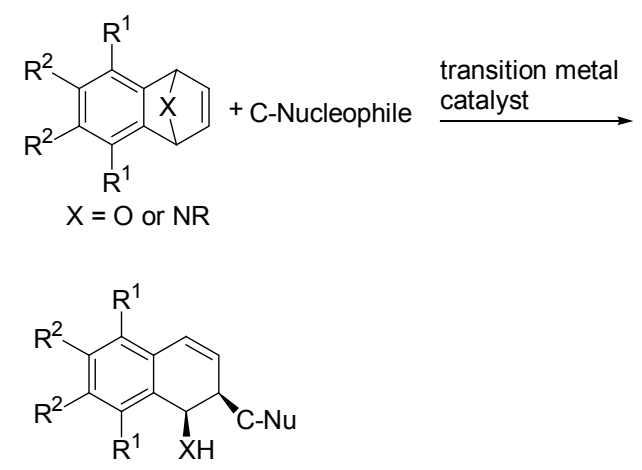

图 1 氧/氮杂苯并降冰片烯与亲核试剂的开环反应

Figure 1 The ring-opening reaction of oxa/azabenzonorbornadienes with carbo-nucleophiles

为发展新的碳亲核开环试剂, 并使所得的氧/氮杂 苯并降冰片烯亲核开环产物能够更好地做进一步的转 化, 末端炔烃这一潜在的碳亲核开环试剂近年来引起了 化学家的重视. 如 2002 年, 郑健鸿 等 ${ }^{[9]}$ 采用 $\mathrm{Ni}($ dppe $) \mathrm{Cl}_{2} 、 \mathrm{ZnCl}_{2}$ 以及 $\mathrm{Zn}$ 粉组成的多组分催化体系, 首次实现了末端炔烃对氧/氮杂苯并降冰片烯的亲核开 环反应，得到了氢化芸骨架上含有炔基的开环产物. 虽 然该开环反应给出了较好的收率, 但催化体系比较复 杂, 加入过量的锌粉使反应在非均相状态下进行, 并给 后处理带来麻烦. 2008 年, Hayashi 等 ${ }^{[10]}$ 报道了手性双膦 配体的铑络合物催化末端炔烃对氮杂苯并降冰片烯的 亲核不对称开环反应, 并以高达 93\%的收率和 $99 \%$ ee 的对映选择性得到相应的开环产物. 遗憾的是, 该手性 铑催化剂的底物适用范围较窄, 仅对具有较大空间位阻 的三烷基硅基乙炔才能得到较好的收率和对映选择性, 且至今仍无氧杂苯并降冰片烯在此催化体系中应用情 况的报道. 因此, 研究和发展简单、新颖、高效、底物 适用范围广的末端炔烃对氧/氮杂苯并降冰片烯的亲核
开环反应仍然是一个具有挑战和重要意义的课题.

最近, 我们课题组对过渡金属催化的末端炔烃与冰 片烯类化合物的不对称环化反应和加成反应非常感兴 趣, 并取得很好的研究进展. 我们发现手性双膦配体, 如具有平面手性的双膦配体 Xylyl-PHANEPHOS, 其铱 络合物能够有效地催化末端炔烃对氧/氮杂苯并降冰片 烯，以及各种冰片烯类化合物的不对称 [2+2]环化反应, 并获得了高达 $89 \%$ 的收率和大于 $99 \%$ ee 的对映选择 性 ${ }^{[11]}$; 而将铱络合物中的平面手性双膦配体 Xylyl-PHANEPHOS 换为轴手性的双膦配体如 SYNPHOS 时, 我们意外发现 $[2+2]$ 环化反应不能发生, 主要得到末端炔烃碳一氢键对冰片烯类化合物中碳一 碳双键的不对称加成反应，即发生不对称催化氢化炔基 化反应 ${ }^{[12]}$. 反应的收率很好，对映选择性也可高达 $97 \%$ $e e$. 因此, 我们拟在此基础上对催化剂的金属前体和 配体进行深入细致的探索, 从而发现和建立末端炔烃对 氧/氮杂苯并降冰片烯等冰片烯类化合物的亲核开环反 应的新型、高效催化体系, 从而为氢化荟衍生物的合成 提供新的有效方法.

鉴于过渡金属钯在碳碳键形成反应中的突出表 现 ${ }^{[13]}$, 我们将研究重点放在钯催化体系的建立上. 经系 统的研究, 我们发现二面角较大的双膦配体 Xantphos 的钯络合物能够有效催化末端炔烃对氧/氮杂苯并降冰 片烯的亲核开环反应，以高达 $91 \%$ 的收率得到相应的炔 基取代二氢萗开环产物. 这也是在温和的反应条件下钯 催化末端炔烃对氧/氮杂苯并降冰片烯 ${ }^{[14]}$ 亲核加成开环 反应的首次发现.

\section{2 结果与讨论}

\section{1 反应条件的篣选及优化}

采用 7-氧杂苯并降冰片烯 $(\mathbf{1 a})(0.3 \mathrm{mmol})$ 和对甲氧 基苯乙炔 (2a) $(0.6 \mathrm{mmol})$ 作为标准底物, 我们对二者间 的开环反应进行了系统的研究. 采用 $\mathrm{Pd}(\mathrm{OAc})_{2}$ 为催化 剂前体、 $\mathrm{PPh}_{3}$ 为配体、 $\mathrm{DCE}(1,2$-二氯乙烷)为溶剂及 70 ${ }^{\circ} \mathrm{C}$ 的加热条件下，7-氧杂苯并降冰片烯(1a)与对甲氧基 苯乙炔(2a)只生成了微量的目标开环产物 3a (Table 1, Entry 1). 将催化剂中的配体改换为 rac-Binap 时, 反应 的结果比 $\mathrm{PPh}_{3}$ 有所改善, 产物 3a 的收率达到 $15 \%$ (Entry 2). 另外三种二面角较大的双膦配体 DPPB, DPPF, DEPphos 使反应的收率得到进一步提高, 分别为 $24 \% ， 29 \%$ 和 $40 \%$ (Entries 3 5). 而二面角更大的 Xantphos 作为配体时, 能够给出最好的反应结果, 开环 产物 3a 的收率达到 51\% (Entry 6). 以 Xantphos 为配体, 我们又对不同的钯化合物作为催化剂前体的情况进行 了考察. Pd(TFA) 2 (三氟醋酸钯)对此反应没有促进作用, 反而使 7-氧杂苯并降冰片烯 (1a)异构化成萗酚 (Entry 7). 采用 $\mathrm{Pd}(\mathrm{acac})_{2},[\mathrm{Pd}(\mathrm{allyl}) \mathrm{Cl}]_{2}, \mathrm{PdBr}_{2}$ 或 $\mathrm{PdI}_{2}$ 为催化剂前 体, 反应的结果与采用 $\mathrm{Pd}(\mathrm{OAc})_{2}$ 时相似, 收率没有显著 
的改变(Entries $8 \sim 11)$. 不过, $\mathrm{PdCl}_{2}$ 却能更好地促进该 反应，并以 $64 \%$ 的最高收率生成目标产物(Entry 12).

表 1 配体和金属钯的篮选

Table 1 Screening of ligands and palladium precursors ${ }^{a}$

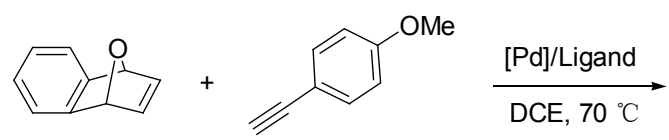

$1 \mathrm{a}$

$2 a$

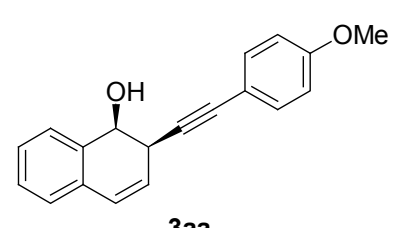

3aa

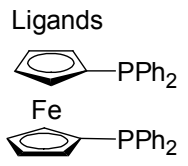

DPPF

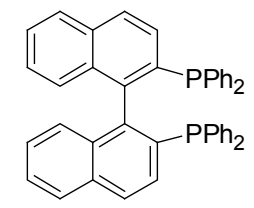

rac-Binap

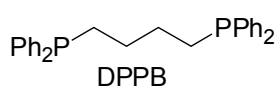
DPPB

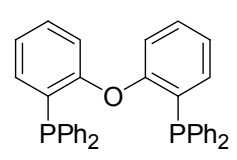

DEPphos

\begin{tabular}{cllcc}
\hline Entry & {$[\mathrm{Pd}]$} & Ligand & Time/h & Yield $^{b} \%$ \\
\hline $1^{\mathrm{c}}$ & $\mathrm{Pd}(\mathrm{OAc})_{2}$ & $\mathrm{PPh}_{3}$ & 24 & Trace \\
2 & $\mathrm{Pd}(\mathrm{OAc})_{2}$ & rac-Binap & 40 & 15 \\
3 & $\mathrm{Pd}(\mathrm{OAc})_{2}$ & DPPB & 24 & 24 \\
4 & $\mathrm{Pd}(\mathrm{OAc})_{2}$ & DPPF & 24 & 29 \\
5 & $\mathrm{Pd}(\mathrm{OAc})_{2}$ & DPEphos & 17 & 40 \\
6 & $\mathrm{Pd}(\mathrm{OAc})_{2}$ & Xantphos & 22 & 51 \\
7 & $\mathrm{Pd}(\mathrm{TFA})_{2}$ & Xantphos & 17 & naphthol \\
8 & $\mathrm{Pd}(\mathrm{acac})_{2}$ & Xantphos & 18 & 45 \\
9 & {$[\mathrm{Pd}(\mathrm{allyl}) \mathrm{Cl}]_{2}$} & Xantphos & 30 & 58 \\
10 & $\mathrm{PdBr}_{2}$ & Xantphos & 30 & 48 \\
11 & $\mathrm{PdI}_{2}$ & Xantphos & 26 & 48 \\
12 & $\mathrm{PdCl}_{2}$ & Xantphos & 26 & 64 \\
\hline
\end{tabular}

${ }^{a}$ Reaction conditions: 1a $(0.3 \mathrm{mmol}), \mathbf{2 a} / \mathbf{1 a} /[\mathrm{Pd}] /$ ligand $=2: 1: 0.05: 0.065$, in DCE $(2 \mathrm{~mL})$ at $70{ }^{\circ} \mathrm{C}$ (oil bath temperature) under Ar for indicated period of time. ${ }^{b}$ Isolated yield by column chromatography. ${ }^{c} \mathbf{2 a} / \mathbf{1 a} /[\mathrm{Pd}] / \mathrm{PPh}_{3}=2$ : $1: 0.05: 0.13$.

采用 $\mathrm{PdCl}_{2} / \mathrm{Xantphos}$ 的配合物作为催化剂, 我们还 对反应条件进行了进一步优化, 结果见表 2. 溶剂实验 表明该催化反应只能在 DCE 中顺利进行, 而在其它溶 剂中(如 DME, DMF, THF, Dioxane, Toluene)则只能生成 微量产物. 我们还对配体用量对反应的影响进行了研 究. 实验结果表明, 当没有配体时, $\mathrm{PdCl}_{2}$ 也能促进 7-氧 杂苯并降冰片烯(1a)与对甲氧基苯乙炔(2a)之间发生反 应, 但产物比较复杂, 而目标开环产物 3a 则生成的很少 (Entry 1). Xantphos 配体的加入能够有效地抑制其它副
反应的发生，而且随着配体用量的逐步增加，反应的收 率也逐步的提高(Entries 2 5). 当配体的用量为 9.2 $\mathrm{mmol} \%$ 时, 催化剂的效果最好, 能使反应的收率达到 $87 \%$. 进一步加大配体的用量不仅无助于催化剂活性的 提高，反而导致反应收率的快速下降(Entries 7,8). 这表 明该反应的活性催化剂很可能是一种 Xantphos 配体与 $\mathrm{PdCl}_{2}$ 所形成的特殊配合物. 该配合物由 Xantphos 配体 中的一个磷原子与钯强配位、另一个磷原子则由于空间 距离的原因而弱配位所形成, 适当过量配体的存在有助 于该配合物的生成，而添加过多的配体则会形成两个 Xantphos 以单膦配体形式与钯配位的稳定配合物, 从而 降低目标反应的速度和收率. 温度对该反应同样具有重 要的影响. 当反应温度降至 $50{ }^{\circ} \mathrm{C}$ 时, 反应的速度会显 著减慢, 即使经过 $4 \mathrm{~d}$ 的加热搅拌, 底物也不能反应完 全, 产物的收率也只有 58\% (Entry 9); 增加反应温度至 $90{ }^{\circ} \mathrm{C}$ 时, 虽然能使反应的速度有所提高, 但产物的收率 却明显降低, 这可能是由于存在一些对温度更为敏感的 副反应所导致(Entry 10).

表 2 反应条件的优化

Table 2 Optimization of reaction conditions ${ }^{a}$
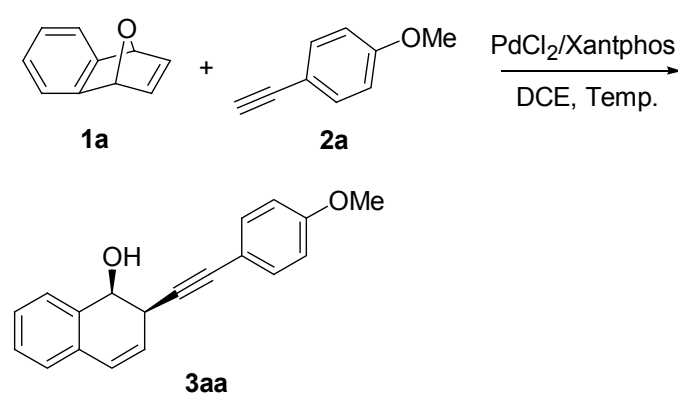

\begin{tabular}{ccccc}
\hline Entry & Temp $/{ }^{\circ} \mathrm{C}$ & Ligand $/ \%$ & Time $/ \mathrm{h}$ & Yield $^{b} / \%$ \\
\hline 1 & 70 & 0 & 24 & Complex \\
2 & 70 & 3.2 & 24 & 34 \\
3 & 70 & 5.5 & 46 & 54 \\
4 & 70 & 6.5 & 26 & 64 \\
5 & 70 & 8.0 & 42 & 68 \\
6 & 70 & 9.2 & 37 & 87 \\
7 & 70 & 10.5 & 46 & 58 \\
8 & 70 & 12 & 60 & 43 \\
$9^{c}$ & 50 & 9.2 & 100 & 58 \\
10 & 90 & 9.2 & 18 & 59 \\
\hline${ }^{a}$ Reaction conditions: 1a $(0.3$ & $\mathrm{mmol}), \mathbf{2 a} / \mathbf{1 a} / \mathrm{PdCl}_{2}=2: 1: 0.05$, in $2 \mathrm{~mL}$
\end{tabular}

${ }^{a}$ Reaction conditions: 1a $(0.3 \mathrm{mmol}), \mathbf{2 a} / \mathbf{1 a} / \mathrm{PdCl}_{2}=2: 1: 0.05$, in $2 \mathrm{~mL}$ DCE at oil bath under Ar for indicated period of time. ${ }^{b}$ Isolated yield by column chromatography. ${ }^{c}$ The reaction was not completed.

\section{2 适用底物范围研究}

在最佳反应条件下，我们对该 $\mathrm{PdCl}_{2} /$ Xantphos 催化 开环反应的底物适用范围进行了研究. 我们首先对具有 不同取代基的各种氧/氮杂苯并降冰片烯与对甲氧基苯 乙炔(2a)的开环反应情况进行了考察，结果见表 3 . 令人 高兴的是，在该钯催化剂作用下，氧杂苯并降冰片烯及 
氮杂苯并降冰片烯都能够顺利地与对甲氧基苯乙炔(2a) 发生开环反应, 并以较好的收率生成相应的开环产物. 在氧杂苯并降冰片烯类底物中, 一般来说, 甲基、甲氧 基等供电子取代基有助于相应底物的反应获得较高的 收率(Entries 2 6), 而吸电子取代基则使反应的收率有 所降低, 比如溴取代的氧杂苯并降冰片烯底物 $\mathbf{1 g}$, 其相 应的开环产物收率只有 53\% (Entry 7). 具有较大空间位 阻菲环结构的氧杂苯并降冰片烯 $1 \mathbf{h}$ 也能顺利参与反应 (Entry 8), 并能获得中等的收率(58\%). Boc 保护的氮杂 苯并降冰片烯也是该催化剂的合适底物, 但由于其反应 活性相对较低, 在与对甲氧基苯乙炔(2a)发生开环反应 时需要较高的反应温度, 从而能够在相对较短的时间内 就使反应结束并获得较好的产物收率(Entries 9～11).

表 3 钯催化各种取代氧/氮杂苯并降冰片烯与对甲氧基苯乙炔的开 环反应

Table 3 Pd-catalyzed ring-opening reaction of substituted aza/oxabenzonorbornadienes with 4-ethynylanisole ${ }^{a}$

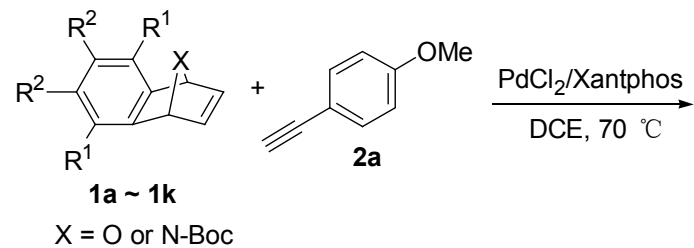<smiles>[R]c1c([R])c([R])c2c(c1[R1])C=CC(C#Cc1ccc(OC)cc1)C2[X]</smiles>

3aa 3ka

\begin{tabular}{|c|c|c|c|c|}
\hline Entry & Alkene & & Time/h & Yield $^{b} / \%$ \\
\hline 1 & & $1 \mathrm{a}$ & 37 & 87 \\
\hline 2 & & $1 b$ & 70 & 87 \\
\hline 3 & & 1c & 50 & 86 \\
\hline 4 & & 1d & 40 & 69 \\
\hline 5 & & $1 \mathrm{e}$ & 36 & 77 \\
\hline
\end{tabular}

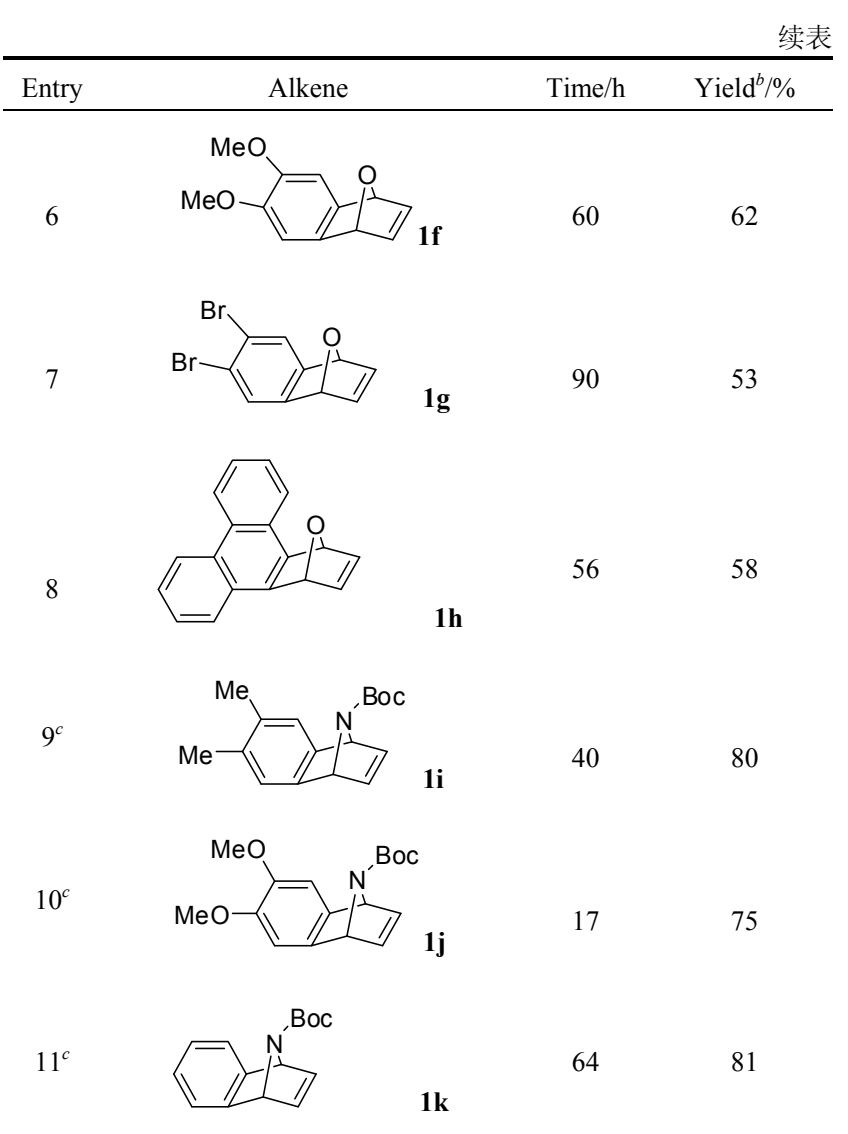

${ }^{a}$ Reaction conditions: $1(0.3 \mathrm{mmol}), \mathbf{2 a} / \mathbf{1} / \mathrm{PdCl}_{2} / \mathrm{Xantphos}=2: 1: 0.05:$ 0.092 , in DCE $(2 \mathrm{~mL})$ at $70{ }^{\circ} \mathrm{C}$ (oil bath temperature) under Ar for indicated period of time. ${ }^{b}$ Isolated yield by column chromatography. ${ }^{c}$ At $90{ }^{\circ} \mathrm{C}$ (oil bath temperature).

随后，我们又考察了苯环上具有不同取代基的芳基 乙炔在该催化反应条件下对氮杂苯并降冰片烯底物 $\mathbf{1 k}$ 的开环反应情况，结果见表 4. 从中可以看出，从最简 单的苯乙炔(Entry 2), 到具有供电子基团的芳基乙炔 (Entries 1，3，5，7)，以及具有吸电子取代基的芳基乙炔 (Entries 4,6), 都能顺利地与氮杂苯并降冰片烯 $1 \mathbf{k}$ 进行 反应，并以较好的收率生成相应的开环产物. 值得一提 的是, $\mathrm{F}, \mathrm{Br}$ 取代基以及活泼的 $\mathrm{CH}_{2} \mathrm{OH}$ 取代基在该钯催 化反应中具有良好的耐受性，从而也使其相应的开环产 物具有更多的衍生化空间(Entries 4,6,8). 可能由于亲 核能力过低的原因，烷基炔烃在该催化条件下的应用并 不成功, 或者只有少量开环产物生成, 或者根本没有反 应发生.

\section{3 结论}

我们建立了钯催化末端炔烃对氧/氮杂苯并降冰片 烯的亲核开环反应的催化体系, 发现以 $\mathrm{PdCl}_{2}$ 为金属前 体和二面角较大的 Xantphos 为配体的催化剂可给出高 达 91\%收率的亲核加成开环产物, 且该催化体系对底物 的适用范围较广，对不同取代或保护的氧/氮杂苯并降 冰片烯都能给出很好的收率。采用手性双膦配体，如 
$(R)$-Tol-Binap, $(R, R)$-Me-Duphos 等, 以期实现钯催化该 反应不对称形式的尝试尚未取得实质性突破, 反应收率 或对映选择性较差; 相应的探索仍在进行当中.

\section{表 4 钯催化下各种芳基乙炔对氮杂苯并降冰片烯的开环反应}

Table 4 Pa-catalyzed ring-opening alkynylation of azabenzonornadiene $1 \mathbf{k}$ with various aryl acetylenes $\mathbf{2 a} \sim \mathbf{2} \mathbf{h}^{a}$<smiles>CC(C)(C)OC(=O)N1C2C=CC(c3ccccc32)C1C(=O)OCc1ccccc1</smiles>

$1 \mathrm{k}$<smiles>[R]c1cccc(C#C[C@H]2C=Cc3ccccc3[C@H]2NC(=O)OCc2ccccc2)c1</smiles>

$3 k a \sim 3 k h$

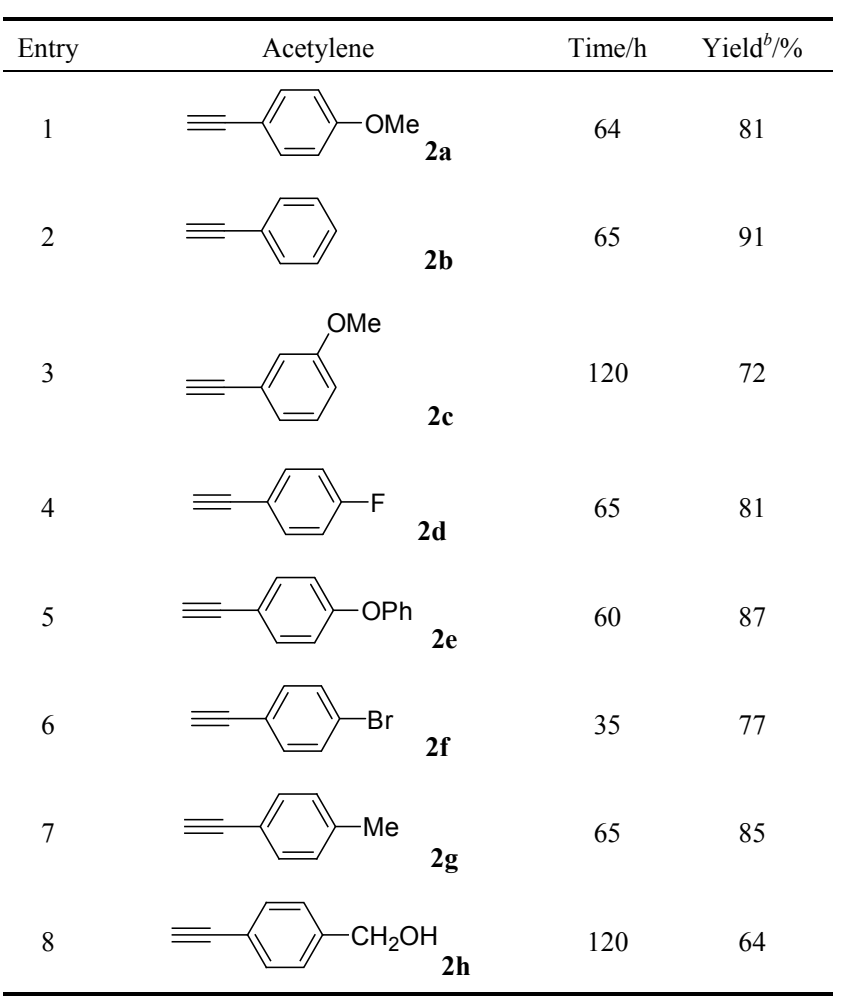

${ }^{a}$ Reaction conditions: $1 \mathbf{k}(0.3 \mathrm{mmol}), \mathbf{2} / \mathbf{1} / \mathrm{PdCl}_{2} / \mathrm{Xantphos}=2: 1: 0.05:$ 0.092 , in DCE $(2 \mathrm{~mL})$ at $90{ }^{\circ} \mathrm{C}$ (oil bath temperature) under Ar for indicated period of time. ${ }^{b}$ Isolated yield by column chromatography.

\section{References}

[1] For selected references: (a) Freeman, J. P.; Michalson, E. T.; D’Andrea, S. V.; Baczynskyj, L.; VonVoigtlander, P. F.; Lahti, R. A.; Smith, M. W.; Lawson, C. F.; Scahill, T. A.; Mizsak, S. A.;
Szmuszkovicz, J. J. Med. Chem. 1991, 34, 1891; (b) Jones, J. H.; Anderson, P. S.; Baldwin, J. J.; Clineschmidt, B. V.; McClure, D. E.; Lundell, G. F.; Randall, W. C.; Martin, G. E.; Williams, M.; Hirshfield, J. M.; Smith, G.; Lumma, P. K. J. Med. Chem. 1984, 27, 1607; (c) Snyder, S. E. J. Med. Chem. 1995, 38, 2395; (d) Kamal, A.; Gayatri, L. Tetrahedron Lett. 1996, 37, 3359; (e) Welch, W. M.; Kraska, A. R.; Sarges, R.; Keo, B. K. J. Med. Chem. 1984, 27, 1508. (f) Snyder, S. E.; Aviles-Garay, F. A.; Chakraborti, R.; Nichols, D. E.; Watts, V. J.; Mailman, R. B. J. Med. Chem. 1995, 38, 2395; (g) Saito, A.; Kayama, Y.; Watanabe, T.; Fukushima, H.; Hara, T. J. Med. Chem. 1980, 23, 1364.

[2] Yang, D.-Q.; Han, Y.-F. Chin. J. Org. Chem. 2006, 26, 1613. (杨定 乔, 韩英锋, 有机化学, 2006, 26, 1613.)

[3] Bos, P. H.; Rudolph, A.; Perez, M.; Fananas-Mastral, M.; Harutyunyan, S. R.; Feringa, B. L. Chem. Commun. 2012, 48, 1748.

[4] (a) Lautens, M.; Renaud, J.; Hiebert, S. J. Am. Chem. Soc. 2000, 122, 1804; (b) Lautens, M.; Hiebert, S. J. Am. Chem. Soc. 2004, 126 1437; (c) Cabrera, S.; Arrayas, R. G.; Alonso, I.; Carretero, J. C. J. Am. Chem. Soc. 2005, 127, 17938; (d) Cabrera, S.; Arrayas, R. G.; Carretero, J. C. Angew. Chem. Int. Ed. 2004, 43, 3944; (e) Bertozzi, F.; Pineschi, M.; Macchia, F.; Arnold, L. A.; Minnaard, A. J.; Feringa, B. L. Org. Lett. 2002, 4, 2703; (f) Li, M.; Yan, X.-X.; Hong, W.; Zhu, X.-Z.; Cao, B.-X.; Sun, J.; Hou, X.-L. Org. Lett. 2004, 6, 2833; (g) Imamoto, T.; Sugita, K.; Yoshida, K. J. Am. Chem. Soc. 2005, 127, 11934; (h) Imamoto, T.; Saitoh, Y.; Koide, A.; Ogura, T.; Yoshida, K. Angew. Chem. Int. Ed. 2007, 46, 8636.

[5] (a) Arrayas, R. G.; Cabrera, S.; Carretero, J. C. Org. Lett. 2003, 5, 1333; (b) Zhang, W.; Wang, L.-X.; Shi, W.-J.; Zhou, Q.-L. J. Org Chem. 2005, 70, 3734; (c) Zhang, W.; Zhu, S.-F.; Qiao, X.-C.; Zhou, Q.-L. Chem. Asian J. 2008, 3, 2105; (d) Millet, R.; Gremaud, L.; Bernardez, T.; Palais, L.; Alexakis, A. Synthesis 2009, 12, 2101.

[6] (a) Millet, R.; Bernardez, T.; Palais, L.; Alexakis, A. Tetrahedron Lett. 2009, 50, 3474; (b) Ladjel, C.; Fuchs, N.; Zhao, J. K.; Bernardinelli, G.; Alexakis, A. Eur. J. Org. Chem. 2009, 29, 4949.

[7] Wu, M.-S.; Jeganmohan, M.; Cheng, C.-H. J. Org. Chem. 2005, 70, 9545.

[8] (a) Murakami, M.; Igawa, H. Chem. Commun. 2002, 390; (b) Lautens, M.; Dockendorff, C.; Fagnou, K.; Malicki, A. Org. Lett. 2002, 4, 1311; (c) Lautens, M.; Dockendorff, C. Org. Lett. 2003, 5, 3695. (d) Zhang, T.-K.; Mo, D.-L.; Dai, L.-X.; Hou, X.-L. Org. Lett. 2008, 10, 3689; (e) Zhang, T.-K.; Mo, D.-L.; Dai, L.-X.; Hou, X.-L. Org. Lett. 2008, 10, 5337.

[9] Rayabarapu, D. K.; Chiou, C.-F.; Cheng, C.-H. Org. Lett. 2002, 4, 1679.

[10] Nishimura, T.; Tsurumaki, E.; Kawamoto, T.; Guo, X.-X.; Hayashi, T. Org. Lett. 2008, 10, 4057.

[11] (a) Fan, B.-M.; Li, X.-J.; Peng, F.-Z.; Zhang, H.-B.; Chan, A. S. C.; Shao, Z.-H. Org. Lett. 2010, 12, 304; (b) Hu, J.; Yang, Q.-J.; Yu, L.; Xu, J.-B.; Liu, S.-S.; Huang, C.; Wang, L.; Zhou, Y.-Y.; Fan, B.-M. Org. Biomol. Chem. 2013, 11, 2294.

[12] (a) Fan, B.-M.; Yang, Q.-J.; Hu, J.; Fan, C.-L.; Li, S.-F.; Yu, L.; Huang, C.; Tsang, W.-W.; Kwong, F.-Y. Angew. Chem., Int. Ed. 2012, 51, 7821; (b) Hu, J.; Yang, Q.-J.; Xu, J.-B.; Huang, C.; Fan, B.-M.; Wang, J.; Lin, C.-Y.; Bian, Z.-X.; Chan, A. S. C. Org. Biomol. Chem. 2013, 11, 814 .

[13] (a) Palladium Reagents and Catalysts, Ed.: Tsuji J., Wiley-VCH, Weinheim, 2003; (b) Li, D.-D.; He, C.-L.; Cai, H.-T; Wang, G.-W. Chin. J. Org. Chem. 2013, 33, 203. (李丹丹, 何程林, 蔡海婷, 王 官武, 有机化学, 2013, 33, 203.); (c) Zhou, L.; Jiang, H.-F.; Huang, J.-M.; Tang, J.-Y. Chin. J. Org. Chem. 2006, 26, 1. (周砧, 江焕峰, 黄精美, 唐金玉, 有机化学, 2006, 26, 1.); (d) Li, J.-H. Acta Chim. Sinica 2004, 62, 341. (李金恒, 化学学报, 2004, 62, 341.)

[14] Ge, G.-C.; Mo, D.-L.; Ding, C.-H.; Dai, L.-X.; Hou, X.-L. Org. Lett. 2012, 14, 5756 . 